\title{
Study on the impact of electric vehicle charging load on nodal voltage deviation
}

\author{
Gang Ma, Linru Jiang, Yixi Chen, Chenyue Dai, Rong Ju \\ School of Electrical \& Automation Engineering, Nanjing Normal University \\ Bancang Street No.78, 210042 Nanjing, China \\ e-mail:nnumg@njnu.edu.cn
}

(Received: 21.09.2016, revised: 07.01.2017)

\begin{abstract}
The promotion and application of electric vehicles will contribute to the solution of several problems, such as energy shortage and environmental pollution, and the achievement of country economy and energy security. But a large-scale vehicle-to-grid system may cause adverse effects in the distribution network operation, the power network planning and such other parts. First, this paper collects the factors that influence the electric vehicle charging load and establishes the EV charging load model with a MonteCarlo method. Then, we analyze the effect that the EV charging load made on the nodal voltage deviation under different permeability based on the IEEE30 node system. At last, this research gets the conclusion that the nodal voltage deviation is closely related to EV permeability, node type and node location. This research conclusion will provide practical guidance to the charging station planning.
\end{abstract}

Key words: distribution network, electric vehicle charging load (EVCL), stochastic load, voltage deviation

\section{Introduction}

Electric Vehicle (EV) has the advantage in energy consumption and emission, its promotion and application will contribute to the solution of several problems, such as energy shortage and environmental pollution, and the achievement of country economy and energy security $[1,2]$. With the development of the new motor, batteries and so on, the electric vehicle industry is gradually entering the stage of rapid development, but a large-scale vehicle-to-grid system may cause adverse effects in the distribution network operation, the power network planning and such other parts [3]. In order to reduce the adverse effects on a power system, it is of practical application value to provide theoretical supports by analyzing these effects based on the research of electric vehicle charging characters.

Domestic and foreign scholars have made some achievements on the related items. Liting Tian proposed a charging load prediction model based on the original load curve, this model can forecast EVs' charging state by analyzing the charge/discharge characteristics of the bat- 
tery and the usage rate of EVs with the Monte-Carlo method [4]. Xueyong Yi established an electric vehicle daily charging load prediction model by analyzing the distribution of the EV charging start time and daily mileage [5]. Jian Wang analyzed the effects that the EV charging load made on the power quality, power system economy, security and stability [6]; he also proposed an optimal control method that includes both centralized and distributed situations and established a coordinated control model [7]. Robert C. Green II considered access point distribution and made stability indicator research on the power system operation with EV [8].

This paper collects the factors that influence the electric vehicle charging load, simulates the electric vehicle charging load with the direct Monte-Carlo method and analyzes the effect that electric vehicles made on the nodal voltage deviation under different permeability based on the IEEE30 node system.

\section{Factors affecting EV charging load}

An EV charging load is mainly affected by permeability, charging characteristics and users' charging behaviour $[9,10]$. Permeability means the proportion of electric vehicles in all cars, charging characteristics includes battery capacity, rated voltage, rated power and so on, users' charging behaviour covers the start charging time, daily mileage and state of charge (SOC). This paper chooses battery electric private vehicles as the research object, the reference model is BYD E6 and its specific parameters are shown in Table 1.

Table 1. Specific parameters of BYD E6

\begin{tabular}{c|c|c|c}
\hline Type & Driving range/km & \multicolumn{2}{|c}{ Battery capacity } \\
\hline $\begin{array}{c}\text { battery electric } \\
\text { vehicle }\end{array}$ & 400 & \multicolumn{2}{|c}{$82 \mathrm{kWh}$} \\
\hline $\begin{array}{c}\text { Charging modes } \\
\text { Mode 1 }\end{array}$ & Charging equipment & Rated voltage & Rated power \\
\hline Mode 2 & wall-mounted charging box & AC $220 \mathrm{~V}$ & $7 \mathrm{~kW}$ \\
\hline Mode 3 & dedicated charging pump & DC $380 \mathrm{~V}$ & $40 \mathrm{~kW}$ \\
\hline
\end{tabular}

The ways for EVs to add energy include battery charging and battery exchanging [11]. The charging equipment, rated voltage and rated power of three charging modes have been shown in Table 1, the standard socket-outlet used in Mode 1 and the wall-mounted charging box used in Mode 2 are both the standard configurations, while the dedicated charging pump used in Mode 3 must be equipped additionally. Mode 2 is more suitable for private EVs with the advantage of low installation cost, low maintenance cost and suitable charging speed, so this paper only considers Mode 2, and its rated power is $7 \mathrm{~kW}$. Assume that the battery charge efficiency $\eta=90 \%$. 
This paper assumes that EV starts charging as soon as it ends its daily trip. The end time can refer to traditional fuel vehicles statistics and its probability distribution meets the normal distribution, the probability density function can be expressed as [12]:

$$
f_{s}=\left\{\begin{array}{l}
\frac{1}{\sigma_{s} \sqrt{2 \pi}} \exp \left[-\frac{\left(x-\mu_{s}\right)^{2}}{2 \sigma_{s}{ }^{2}}\right] \quad\left(\mu_{s}-12\right)<x \leq 24 \\
\frac{1}{\sigma_{s} \sqrt{2 \pi}} \exp \left[-\frac{\left(x+24-\mu_{s}\right)^{2}}{2 \sigma_{s}{ }^{2}}\right] \quad 0<x \leq\left(\mu_{s}-12\right)
\end{array},\right.
$$

where: $\mu_{s}$ stands for the expected value, $\mu_{s}=17.6 ; \sigma_{s}$ stands for the standard deviation, $\sigma_{s}=3.4$. EV charging start time distribution is simulated by MATLAB as shown in Fig. 1.

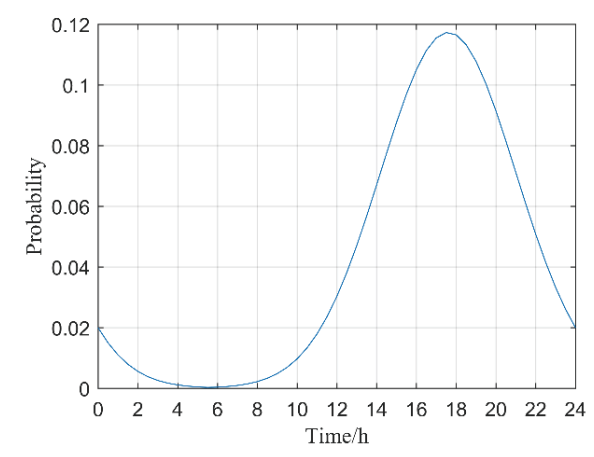

Fig. 1. EV charging start time distribution

Daily mileage $r / \mathrm{kM}$ is an important parameter that determines the daily power consumption, it is independent of the EV type and can refer to traditional vehicle data from National Household Travel Survey (NHTS), its probability distribution meets the logarithmic normal distribution, the probability density function can be expressed as [11]:

$$
f_{D}(r)=\frac{1}{r \sigma_{D} \sqrt{2 \pi}} \exp \left[-\frac{\left(\ln r-\mu_{D}\right)^{2}}{2 \sigma_{D}^{2}}\right],
$$

where: $\mu_{D}$ stands for the logarithmic average of $r, \mu_{D}=3.2 ; \sigma_{D}$ stands for the logarithmic standard deviation of $r, \sigma_{D}=0.88, \mathrm{EV}$ changing power distribution can be obtained by combining daily mileage, charging power with charging start time.

\section{EV charging load calculation}

EV power demand $E / \mathrm{kM}$ is determined by daily mileage $r$ and can be expressed as the following equation according to Table 1. 


$$
E=r \times 82 / 400 .
$$

Charging power under the conventional charging mode is $7 \mathrm{~kW}$, so charging time for every vehicle $T / \mathrm{h}$ can be expressed as:

$$
T=E /(7 \times \eta) .
$$

Nowadays, the common methods used in EV charging power calculation include the statistical simulation method, the Monte-Carlo simulation method and the calculation method based on the Diffusion Theory, this paper selects the direct Monte-Carlo simulation method. We sample charging start time and daily mileage to simulate the usage of every EV, then stack all the EVs' charging loads and obtain an EV charging load curve. The process of EV load curve simulation is shown in Fig. 2.

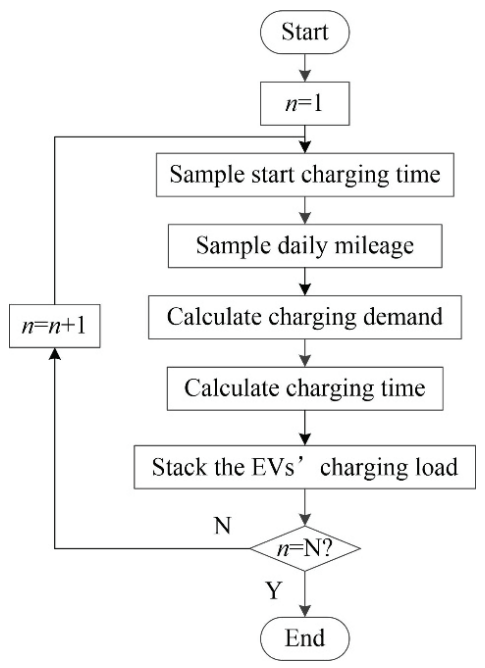

Fig. 2. Charging load calculation

Take 20000 EVs for example, the charging load curve is shown in Fig. 3.

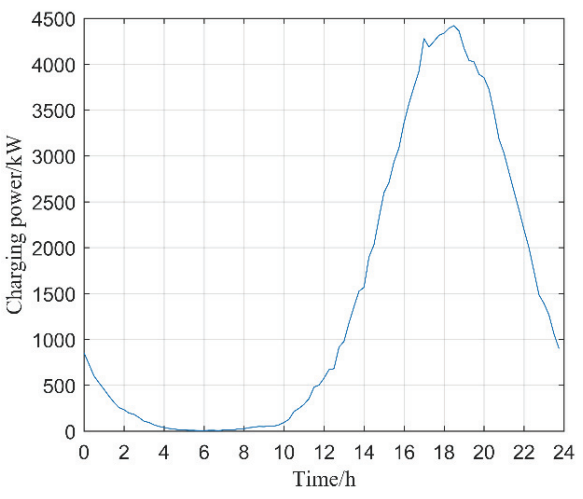

Fig. 3. The charging load curve of $20000 \mathrm{EVs}$ 
The EV charging load is closely linked to users' daily trip, EV leaves the grid when drivers go out in the morning and accesses to the grid when the daily trip ends, so the charging load curve reflects the driving rules directly. The peak/valley charging load situation exists according to Fig. 3, the peak time around 6:00 approximately dovetails the time people go for work and the valley time around 18:00 approximately dovetails the time people go off work. Between 6:00 and 18:00, charging load is rising until it gets the maximum since most people ends the daily trip. Then, the charging load decreases since part of EVs finish charging, the total charging load is almost zero at 4:00.

The EV peak/valley charging load situation is similar to the power system, when a large number of EVs access to the distribution network, the superposition of two partial loads is highly possible to increase power fluctuation and impact the power quality [13]. To explore the effect the EV charging load made on the voltage deviation, this paper analyzes the power system load flow under different permeability of EV.

\section{Power flow analysis}

After EVs accessing to the grid, the superposition of the EV charging load and the power system load is highly possible to increase power fluctuation and impact the power quality. Power flow calculation is the most basic part in power system design and operation, it is intended to collect nodal voltages and power loads, analyze the power flow distribution and figure out stability calculation and failure analysis. In order to analyze the effect the EV charging load made on the voltage deviation, this paper selects the Newton-Raphson method to obtain correction equations and corrected state variables through iteration, then the new variable value meeting the convergence conditions can be achieved to analyze the voltage deviation [14-16].

The nodal complex power can be expressed in a Cartesian coordinate system:

$$
\widetilde{S}_{i}=P_{i}+j Q_{i}=\dot{U}_{i} I_{i}^{*}=\dot{U}_{i} \sum_{j=1}^{n} Y_{i j}^{*} U_{j}^{*},
$$

where the voltage $U_{i}=e_{i}+j f_{i}$ and the nodal admittance $Y_{i j}=G_{i j}+j B_{i j}$. The equation of $P_{i}$ and $Q_{i}$ can be expanded as:

$$
\left.\begin{array}{c}
P_{i}=e_{i} \sum_{j=1}^{n}\left(G_{i j} e_{j}-B_{i j} f_{j}\right)+f_{i} \sum_{j=1}^{n}\left(G_{i j} e_{j}+B_{i j} e_{j}\right) \\
Q_{i}=f_{i} \sum_{j=1}^{n}\left(G_{i j} e_{j}-B_{i j} f_{j}\right)-e_{i} \sum_{j=1}^{n}\left(G_{i j} f_{j}+B_{i j} e_{j}\right)
\end{array}\right\} .
$$

Nodes in the power system is divided into three types: $P Q, P V$ and slack node. The active and reactive power injections of $P Q$ nodes are certain, the unbalance can be expressed as: 


$$
\left.\begin{array}{rl}
\Delta P_{i} & =P_{i}-\sum_{j=1}^{n}\left[e_{i}\left(G_{i j} e_{j}-B_{i j} f_{j}\right)+f_{i}\left(G_{i j} f_{j}+B_{i j} e_{j}\right)\right] \\
\Delta Q_{i} & =Q_{i}-\sum_{j=1}^{n}\left[f_{i}\left(G_{i j} e_{j}-B_{i j} f_{j}\right)-e_{i}\left(G_{i j} f_{j}+B_{i j} e_{j}\right)\right]
\end{array}\right\} .
$$

The active power injections and voltages of $P V$ nodes are certain, the unbalance value can be expressed as:

$$
\left.\begin{array}{c}
\Delta P_{i}=P_{i}-\sum_{j=1}^{n}\left[e_{i}\left(G_{i j} e_{j}-B_{i j} f_{j}\right)+f_{i}\left(G_{i j} f_{j}+B_{i j} e_{j}\right)\right] \\
\Delta U_{i}^{2}=U_{i}^{2}-\left(e_{i}^{2}+f_{i}^{2}\right)
\end{array}\right\} .
$$

Arrange both sides of the unbalance equations into column vector form, which means $\Delta f=J \Delta x$, where, $J$ stands for a Jacobi matrix. The elements in this matrix can be expressed as:

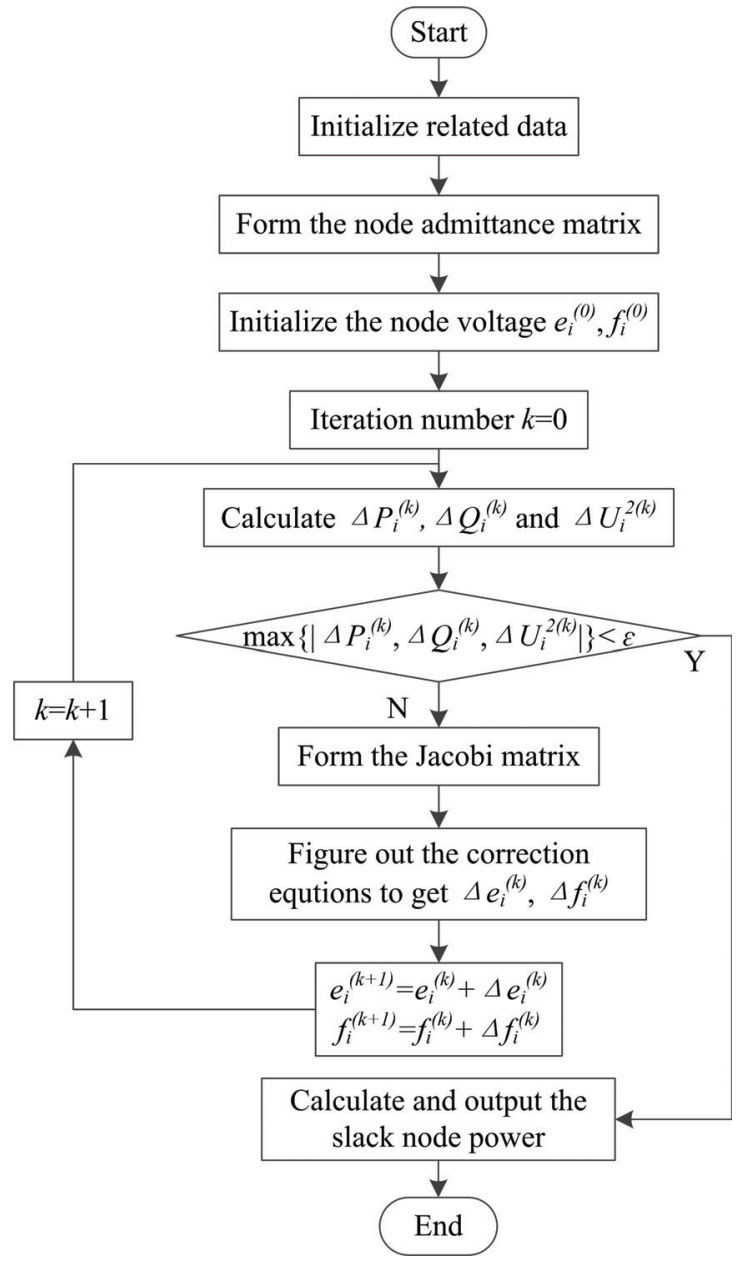

Fig. 4. Power flow calculation 


$$
\begin{aligned}
& H_{i j}=\frac{\partial P_{i}}{\partial f_{j}} \quad N_{i j}=\frac{\partial P_{i}}{\partial e_{j}} \\
& \left.J_{i j}=\frac{\partial Q_{i}}{\partial f_{j}} \quad L_{i j}=\frac{\partial Q_{i}}{\partial e_{j}}\right\} . \\
& R_{i j}=\frac{\partial U_{i}^{2}}{\partial f_{j}} S_{i j}=\frac{\partial U_{i}^{2}}{\partial e_{j}}
\end{aligned}
$$

The specific iterative process is shown in Fig. 4.

The unbalance meeting $\max \left\{\left|\Delta P_{i}^{(k)}, \Delta Q_{i}^{(k)}, \Delta U_{i}^{2(k)}\right|\right\}<\varepsilon$ can be obtained through iteration after all the data initialized, and the corrected voltage value can be expressed as:

$$
\left.\begin{array}{rl}
e_{i}^{(k+1)} & =e_{i}^{(k)}+\Delta e_{i}^{(k)}, f_{i}^{(k+1)}=f_{i}^{(k)}+\Delta f_{i}^{(k)} \\
U_{i}^{(k+1)} & =U_{i}^{(k)}+\Delta U_{i}^{(k)}, \sigma_{i}^{(k+1)}=\sigma_{i}^{(k)}+\Delta \sigma_{i}^{(k)}
\end{array}\right\} .
$$

Slack node power can be figured out by Equation (10), and line power can be figured out by Equation (11)

$$
\widetilde{S}_{n}=\dot{U}_{n} \sum_{j=1}^{n} Y_{n i}^{*} U_{i}^{*}=P_{n}+j Q n
$$

\section{The effect EV made on the voltage deviation}

Large-scale EV charging will cause the voltage deviation which will do harm to people's life and work, shorten the service life of electric power devices and make a negative impact on the power system. The power system is complex and fluctuating so the voltage deviation cannot be avoided, but the voltage deviation should be controlled in suitable range from the view of technical and economical operation. This paper considers the randomness of the EV start charging time and daily mileage completely, and researches the effect that EV charging load made on the voltage deviation based on the IEEE-30 system shown in Fig. 5.

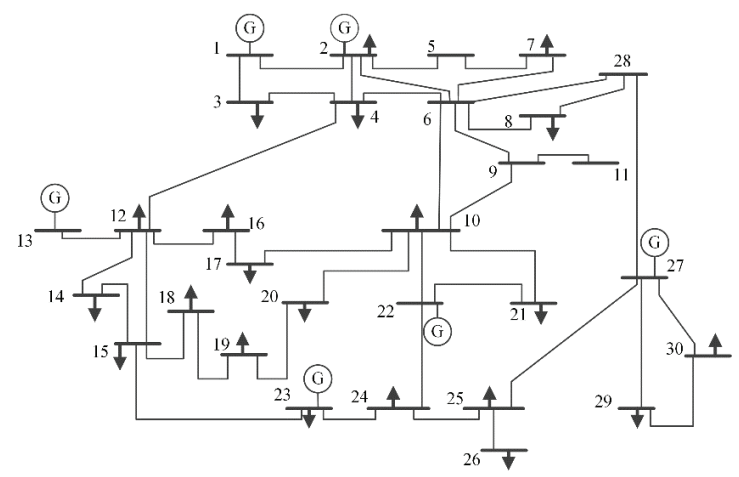

Fig. 5. IEEE-30 node system 
Voltage deviation is defined as the difference between the actual voltage $U / \mathrm{kV}$ and the rated voltage $U_{N} / \mathrm{kV}$, generally expressed in percentage term:

$$
U \%=\frac{U-U_{N}}{U_{N}} \times 100 \%
$$

According to Equation (12), voltage deviation is only related to the actual voltage which is related to flow distribution. After EV accessing to the grid, the flow distribution is affected by the EV permeability, EV charging method and users' driving rules.

The farther the node from the power supply is, the bigger the line loss is, so we can speculate that the nodal voltage deviation is related to the distance between the EV charging access point and the power supply. Assume that EVs access to nodes in the power system randomly and evenly, we stack EV charging load under different EV permeability which is simulated in Section 3 on each node and calculate power flow based on the Newton-Raphson method to get each nodal voltage deviation. The specific process is shown in Fig. 6.

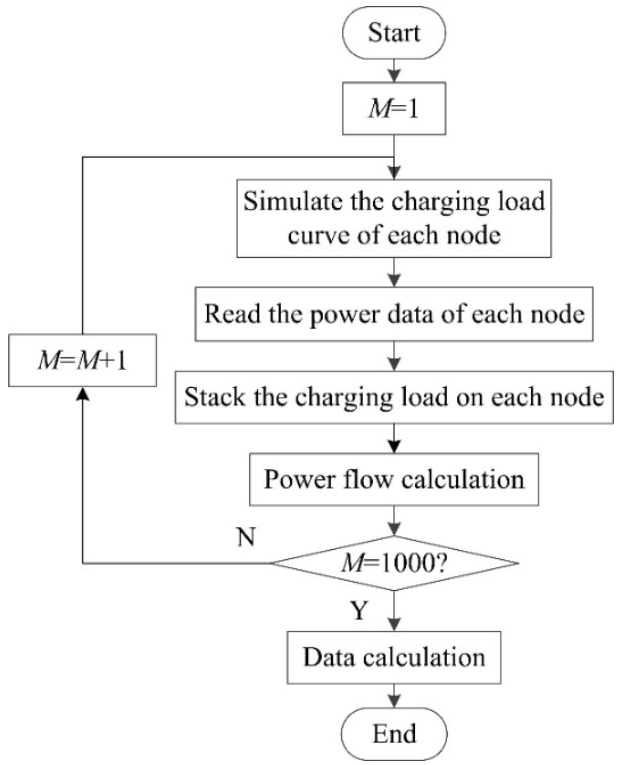

Fig. 6. The effect that EV made on the power system analysis

Take the EV permeability equals $10 \%$ for example, the nodal voltage per-unit values are shown in Fig. 7.

Among them, the voltage deviation of slack node 1 is always 0 ; node 2, 13, 22, 23 and 27 are connected to generators, the actual voltage values of these nodes are stabilized between $95 \%$ and $105 \%$ of the rated voltage value. The distance from node 16 to the power supply node 13 is farther than the distance from node 12 to node 13 . After analysis, we can draw the conclusion that the voltage deviation of the farther node from the power supply node will be bigger. 


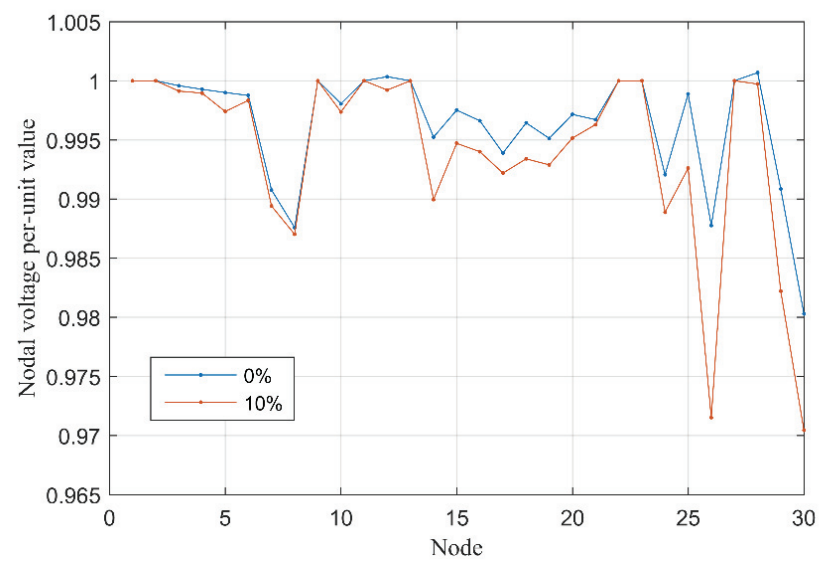

Fig. 7. Nodal voltage per-unit values when EV permeability equals $10 \%$

The nodal voltage per-unit values under different EV permeability are shown in Fig. 8.

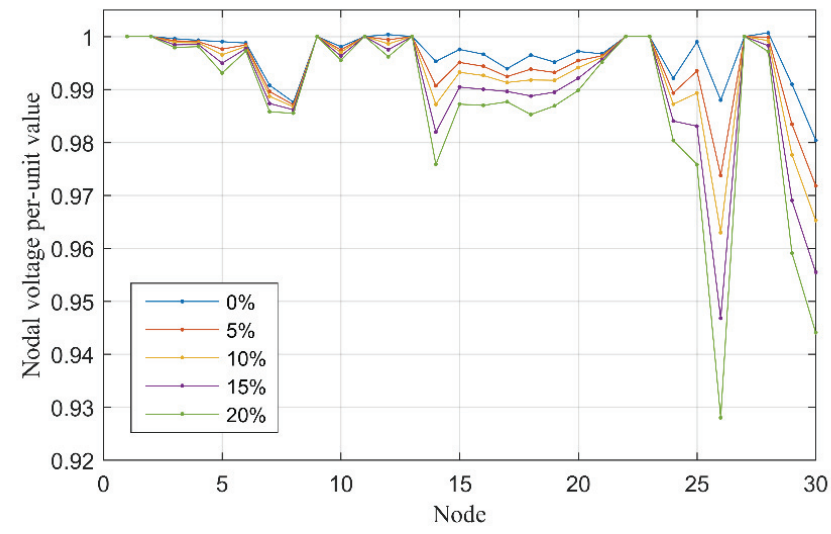

Fig. 8. Nodal voltage per-unit values under different EV permeability

Take node 26 for example and its voltage per-unit value is 0.9881 when no EV access to the power system; the voltage per-unit value drops to 0.9280 when the EV permeability rises to $20 \%$, nodes also greatly affected by the EV charging load include node 29 and 30 . To ensure the reliable operation of the power system, we should avoid EVs accessing this types of nodes in the power network planning.

In the power system with EVs, the nodal voltage deviation is related to the EV permeability and the load access point according to Fig. 7 and Fig. 8. The nodal voltage deviation increases while the EVs permeability raises, and the farther nodes from the power supply node will make a bigger difference between the actual voltage and the rated voltage and be affected by the EV charging load more easily. 


\section{Conclusion}

The promotion and application of electric vehicles will contribute to the solution of several problems, such as energy shortage and environmental pollution, and the achievement of country economy and energy security. But a large-scale vehicle-to-grid system may cause adverse effects in the distribution network operation, the power network planning and such other parts. First, this paper collects the factors that influence the electric vehicle charging load and establishes the EV charging load model with a Monte-Carlo method. Then, we analyze the effect that the EV charging load made on the nodal voltage deviation under different permeability based on the IEEE30 node system. At last, this research gets the conclusion that the nodal voltage deviation is closely related to EV permeability, a node type and node location. This research conclusion will provide practical guidance to the charging station planning.

Several factors will limit the allowed EV permeability except the node voltage deviation, such as transformer capacity, so we can't get conclusion the highest allowed EV permeability for each node. Moreover, this paper assumes that the EV charging load is distributed to each node evenly, it needs more consideration on the spatial distribution of the electric vehicle charging load. We set these two parts as the research direction in the future, and expect to draw more valuable research conclusions.

\section{Acknowledgements}

This work was supported by the National Natural Science Foundation of China under grant number 51607093; Natural Science Foundation of Jiangsu Province under grant number BK20141452; Natural Science Research of Jiangsu Higher Education Institutions of China under grant number 14KJB470006; and High level talents in Nanjing Normal University of China under grant number 2014111XGQ0078.

\section{References}

[1] Shi X., Li X., Yang J., Research on Carbon Reduction Potential of Electric Vehicles for Low-Carbon Transportation and Its Influencing Factors, Environmental Science, vol. 34, no. 1, pp. 385-394 (2014).

[2] Jouybari-Moghaddam H., Alimardani A., Hosseinian S.H., Influence of electric vehicle charging rates on transformer derating in harmonic-rich battery charger applications, Archives of Electrical Engineering, vol. 61, no. 4, pp. 483-497 (2012).

[3] Hu Z., Song Y., Xu Z. et al., Impacts and Utilization of Electric Vehicles Integration Into Power Systems, Proceedings of the CSEE, vol. 32, no. 4, pp. 1-10 (2012).

[4] Tian L., Shi S., Jia Z., A Statistical Model for Charging Power Demand of Electric Vehicles, Power System Technology, vol. 34, no. 11, pp. 126-130 (2010).

[5] Ai X., Gu J., Xie D., Jin Z., Ai Q., Forecasting Method for Electric Vehicle Daily Charging Curve, Proceedings of the CSU-EPSA, vol. 25, no. 6, pp. 55-61 (2013).

[6] Wang J., Studies on the Impacts of Electric Vehicles Charging on the Power System and Coordinated Charging, PhD Thesis, Shandong University (2013).

[7] Wang J., Wu K., Liu Z. et al., Impact of electric vehicle charging on distribution network load and coordinated control, Electric Power Automation Equipment, vol. 33, no. 8, pp. 47-52 (2013).

[8] Green II R.C., Wang L., Alam M., The impact of plug-in hybrid electric vehicles on distribution networks, A review and outlook, Renewable and Sustainable Energy Reviews, vol. 5, pp. 544-533 (2011). 
[9] Xiao X., Zhou B., Fu J. et al., Energy supply schemes for low penetration of electric vehicles and optimal planning for energy supply facilities based on LCC, Power System Protection and Control, vol. 41, no. 20, pp. 53-60 (2013).

[10] Sweeting W.J., Hutchinson A.R., Savage S.D., Factors affecting electric vehicle energy consumption, International Journal of Sustainable Engineering, vol. 4, no. 3, pp. 192-201 (2011).

[11] Bauer P., Zhou Y., Doppler J., Stembridge N., Charging of Electric Vehicles and Impact on the Grid, 2010 13th International Symposium, vol. 2, pp. 121-127 (2010).

[12] Pieltain F.L., Gome S.R.T., Roman T. et al., Assessment of the impact of plug-in electric vehicles on distribution networks, IEEE Trans on Power Systems, vol. 26, no. 1, pp. 206-213 (2010).

[13] Guolong Y., Research on Power Qualiry Analysis and Improvement on Charging Equipment of Electric Vehicle, PhD Thesis, North China Electric Power University (2011).

[14] Tinney W.F., Hart C.E., Power flow solution by Newton's method, IEEE Transactions on Power Apparatus and Systems, vol. 86, pp. 1449-1460 (1967).

[15] Liu C., Zhang B., Revised Algorithm for AC/DC Power Flow and Its Robustness Analysis, Proceedings of the CSEE, vol. 29, no. 19, pp. 57-62 (2009).

[16] Qin Z., Hou Y., Wu F.F., Practical Model for Large-scale AC-DC System Power Flow Calculation, Proceedings of the CSEE, vol. 31, no. 10, pp. 95-101 (2011). 